\title{
Association of Abnormal Preoperative Laboratory Values with Survival After Radical Nephrectomy for Clinically Confined Clear Cell Renal Cell Carcinoma
}

\author{
James S. Magera Jr, Bradley C. Leibovich, Christine M. Lohse, Shomik Sengupta, John C. \\ Cheville, Eugene D. Kwon, and Michael L. Blute \\ From the Departments of Urology, Health Sciences Research, and Laboratory Medicine and \\ Pathology, Mayo Clinic, Rochester, Minnesota
}

\section{Abstract}

Objectives-To determine whether preoperative laboratory values are independently associated with death from clinically confined clear cell renal cell carcinoma (RCC) after radical nephrectomy.

Methods-We identified 1707 patients with clinically confined (pNx/pN0, pM0), unilateral, sporadic clear cell RCC treated with radical nephrectomy between 1970 and 2002. Associations of abnormal preoperative laboratory values including hypercalcemia, anemia, elevated erythrocyte sedimentation rate (ESR), and elevated alkaline phosphatase with death from RCC were evaluated using Cox proportional hazards regression models, both univariately and multivariately by adjusting for known prognostic features of the 2002 primary tumor classification, tumor size, nuclear grade, and coagulative tumor necrosis.

Results-At last follow-up, 1009 patients had died, including 425 who died from RCC at a median of 3.0 years after surgery (range, 0 to 26 years). Even after adjusting for known prognostic features, $9 \%$ of patients with preoperative hypercalcemia exhibited significantly increased likelihood of dying from RCC compared with patients with normal or lower levels of serum calcium (relative ration [RR] $1.64 ; P=0.002$ ). Similarly, preoperative anemia (35\% of patients; RR $1.27 ; P=0.026)$ and elevated ESR (44\% of patients; RR 1.66; $P=0.003$ ) portended an increased risk of death from RCC even after multivariate adjustment.

Conclusions-Abnormal preoperative laboratory values including hypercalcemia, anemia, and elevated ESR are independently associated with increased risk of cancer-specific death from clinically confined clear cell RCC. Consideration of these variables in future models may improve prognostic accuracy. We believe these factors should be routinely assessed and included in prospective studies of outcome in RCC patients.

An estimated 36,000 patients will have been diagnosed with renal cell carcinoma (RCC) in $2005 .{ }^{1}$ Despite surgical extirpation of clinically localized RCC, approximately $30 \%$ will have developed metastatic disease. ${ }^{2}$ This high rate of disease progression has led many investigators to develop prognostic models for prediction of disease progression and cancer-specific death to aid in formulation of surveillance strategies and postoperative patient counseling. Various preoperative laboratory values, including anemia and elevated erythrocyte sedimentation rate (ESR), have been shown to be predictive of cancer-specific death and designated as prognostic markers by the Union Internationale Contre le Cancer (UICC) and American Joint Committee on Cancer (AJCC) consensus report. ${ }^{3,4}$ These features have been validated as predictors of

Reprint requests: Dr. Bradley C. Leibovich, M.D., Department of Urology, Mayo Clinic, 200 First St. SW, Rochester, MN 55905. leibovich.bradley@mayo.edu. 
outcome in a prognostic model for patients with metastatic RCC who concomitantly present with abnormal laboratory values (hypercalcemia, anemia, and elevated lactate dehydrogenase). ${ }^{5}$ In contrast, comprehensive studies of preoperative laboratory values as potential prognostic indices for patients undergoing extirpative surgery for presumably localized RCC have not been systematically conducted. ${ }^{2,6,7}$

More specifically, most studies supporting the prognostic ability of preoperative laboratory values have been composed of small cohorts and not confirmed by larger contemporary series. In fact, one recent large retrospective study failed to reveal associations of hypercalcemia and anemia with cancer-specific death from RCC after multivariate analysis controlling for stage, grade, and ECOG performance status. ${ }^{8}$ In addition, previous studies have clustered chromophobe, papillary, and clear cell RCC subtypes into a single pathologic entity during analysis, despite the known histologic, genetic, and prognostic differences of these RCC subtypes. ${ }^{9-11}$ Combined analysis of localized and metastatic RCC patients has further confounded the interpretation of prior studies analyzing the utility of preoperative laboratory values as indicators of prognosis. ${ }^{8}$ Hence, to address these limitations, we examined a large cohort of patients with clinically confined ( $\mathrm{pNx} / \mathrm{pN} 0, \mathrm{pM} 0)$, clear cell RCC and long-term follow-up to assess whether preoperative laboratory values might serve as independent prognosticators of outcome for patients with RCC.

\section{Material and Methods}

\section{Patient Selection}

Upon approval from the Mayo Clinic Institutional Review Board, we identified 1707 patients treated with open radical nephrectomy for clinically confined ( $\mathrm{pNx} / \mathrm{pN} 0, \mathrm{pM} 0)$, unilateral, sporadic clear cell RCC between 1970 and 2002 from the Mayo Clinic Nephrectomy Registry. Associations with outcome were evaluated for patients with clear cell RCC only given the documented differences in outcome and associations with outcome by RCC histological subtype. ${ }^{9-11}$

\section{Pathologic Features}

The pathologic features studied included tumor size, the 2002 primary tumor classification, nuclear grade, and histological coagulative tumor necrosis. ${ }^{12}$ The microscopic slides from all specimens were reviewed by a single urologic pathologist (J.C.C.) without knowledge of patient outcome. Histological subtype was classified according to the UICC, AJCC, and Heidelberg guidelines. ${ }^{13,14}$ The pathological features studied are shown in Table 1.

\section{Abnormal Preoperative Features}

The preoperative laboratory values evaluated included serum calcium, hemoglobin, serum alkaline phosphatase (ALP), and erythrocyte sedimentation rate (ESR). These laboratory values were selected for analysis based on the UICC/AJCC consensus that they have prognostic value. ${ }^{3,4}$ To minimize potential bias introduced by selection of abnormal value thresholds a posteriori, ${ }^{4}$ we used our institution's gender-specific thresholds for hypercalcemia, anemia, and elevated ESR (Table 2). The threshold for ALP was based on previous literature. ${ }^{5}$

\section{Statistical Methods}

We estimated cancer-specific survival using the Kaplan-Meier method. The duration of followup was calculated from the date of surgery to the date of death or last follow-up. Cause of death was determined from the death certificate or physician correspondence and deaths from causes other than RCC were censored. We assessed the associations of the abnormal preoperative laboratory values with death from RCC using Cox proportional hazards regression models and 
summarized with risk ratios (RR) and 95\% confidence intervals (CI). These associations were evaluated univariately and after adjusting for the Mayo Clinic Clear Cell SSIGN (Stage, Size, Grade, and Necrosis) score. ${ }^{7}$ The SSIGN score was used as a means of adjusting for known predictors of death from clear cell RCC. Although other scoring systems for RCC with a high degree of prognostic ability have been published, we chose to use the SSIGN score because it was specifically developed for clear cell RCC and it had obvious relevance to our patient sample. In addition, a separate multivariate analysis controlling for the 2002 primary tumor classification, nuclear grade, and ECOG performance status was performed. ${ }^{6}$ We performed statistical analyses using the SAS software package (SAS Institute, Cary, NC). All tests were two-sided and $P$ values less than 0.05 were considered statistically significant.

\section{Results}

Table 1 lists clinical and pathological features for the 1707 patients with clinically confined clear cell RCC studied. The median SSIGN score was 3 (range, 0 to 9). ${ }^{15}$ At last follow-up, 1009 patients had died, including 425 patients who died from their disease at a median of 3.0 years after surgery (range, 0 to 26 years). Among the 698 patients who were still alive at last follow-up, the median duration of follow-up was 8.9 years (range, 0 to 34 years). The estimated cancer-specific survival rates (standard error, number still at risk) at 5 and 10 years after surgery were $82.1 \%(1.0 \%, 1007)$ and $72.8 \%(1.3 \%, 554)$, respectively.

Table 2 shows the univariate and multivariate associations of the abnormal preoperative laboratory values studied with death from RCC. Hypercalcemia, anemia, elevated ESR, and elevated ALP were noted in 9\% (119 of 1390), 35\% (584 of 1695), 44\% (297 of 677), and 81\% (1163 of 1444), respectively. Even after adjusting for the SSIGN score, patients with clinically confined disease and hypercalcemia were significantly more likely to die from RCC compared with patients with normal or lower levels of serum calcium (RR 1.64; 95\% CI 1.20 to 2.25; $P=0.002$ ). By examining patients with clinically confined disease, we were able to minimize the potential confounding influence of bone metastases on hypercalcemia. In addition, patients with anemia at presentation were $27 \%$ more likely to die from RCC compared with patients with normal or elevated hemoglobin, even after adjusting for the SSIGN score (RR 1.27; $95 \%$ CI 1.03 to $1.57 ; P=0.026)$. Also, patients with elevated ESR and clinically confined clear cell RCC were $66 \%$ more likely to die from RCC after adjusting for the SSIGN score (RR 1.66; $95 \%$ CI 1.19 to $2.33 ; P=0.003$ ). Finally, patients with elevated ALP did not appear to have an increased risk of death from RCC, either univariately or multivariately.

We performed a separate multivariate analysis controlling for primary tumor classification, nuclear grade, and ECOG performance status on the subset of 1122 patients with ECOG performance status available in our institutional registry. Hypercalcemia (RR 1.68; 95\% CI 1.21 to $2.34 ; P=0.002$ ), anemia (RR $1.44 ; 95 \%$ CI 1.16 to $1.80 ; P=0.001$ ), and elevated ESR (RR 2.04; $95 \%$ CI 1.40 to $2.96 ; P<0.001$ ) remained independently associated with death from RCC.

\section{Comment}

A considerable number of studies examining the prognostic ability of abnormal preoperative laboratory values in patients with RCC have been reported in the literature. In fact, sufficient evidence has now been presented to prompt the UICC/AJCC consensus conference to establish anemia and elevated ESR as useful prognostic markers for patients with RCC. ${ }^{3,4}$ In addition, serum calcium and ALP have been suggested to provide prognostic information for patients with metastatic RCC. ${ }^{3,4}$ Many of the studies that were used to establish UICC/AJCC guidelines, however, failed to take into consideration differences in RCC histologic subtypes and examined relatively small cohorts of patients with both localized as well as advanced forms 
of RCC. Moreover, a recent retrospective study failed to find associations between anemia and hypercalcemia with death from RCC. ${ }^{8}$ Thus, the prognostic potential of preoperative blood tests for patients with RCC remains somewhat controversial. Hence, in an effort to better elucidate the prognostic value of clinical blood tests (chemistries and blood counts), especially for patients with presumed organ-confined RCC, we studied associations between various preoperative laboratory values with death from $\mathrm{RCC}$ in a large cohort of patients who were consecutively treated at our institution with radical nephrectomy for clear cell RCC between 1970 and 2002. In our review, we observe that preoperative hypercalcemia, anemia, and elevated ESR are associated with an increased risk of cancer-specific death in patients with clinically confined RCC, even after multivariate adjustment for other well-known predictors of RCC tumor aggressiveness (Table 2).

Hypercalcemia associated with RCC can be classified into metastatic and nonmetastatic forms. 16,17 The mechanism of metastatic hypercalcemia involves the local interaction of osseus RCC metastatic deposits in bone leading to osteoclast activation and consequent bone resorption. By restricting our analysis to patients with clinically confined RCC, the influence of hypercalcemia resulting from metastatic disease was minimized. Nonmetastatic hypercalcemia is considered to be a paraneoplastic process and may involve the elaboration of parathyroid hormone-related peptide by the tumor, which mimics the hypercalcemic effect of endogenous parathyroid hormone or other humoral mediators such as osteoclast activating factor, transforming growth factor alpha, interleukin 1 , and tumor necrosis factor. ${ }^{16,17}$

Hypercalcemia has been found to portend a poor prognosis for patients with metastatic RCC. 5,17 In contrast, previous studies have failed to identify an association of hypercalcemia with death from RCC in patients with clinically confined disease. The definition of hypercalcemia tends to differ among studies, making direct comparisons of such studies difficult. $5,8,17$ Nevertheless, hypercalcemia in RCC patients has been reported to range from $3 \%$ to $16 \% .^{17}$ Consistent with this, serum calcium was elevated in 119 of the 1390 (9\%) RCC patients we studied. Contrary to other studies and the UICC/AJCC consensus statement, ${ }^{3}$ however, our data suggest that the association of hypercalcemia with death from RCC is not singularly limited to patients with metastatic disease. Instead, our study indicates that hypercalcemia is independently associated with cancer-specific death in patients with clinically confined clear cell RCC after adjusting for primary tumor classification, tumor size, nuclear grade, coagulative tumor necrosis, and ECOG performance status.

Anemia is often observed in patients with newly diagnosed RCC, even before therapeutic intervention. ${ }^{16}$ Several mechanisms whereby malignancy induces anemia have been suggested, including impairment of iron metabolism and elevations in inflammatory cytokine levels contributing to reductions in renal erythropoietin production. ${ }^{18}$ Anemia may also contribute to tumor hypoxia, which has been implicated in the emergence of more aggressive tumor growth for many maligancies ${ }^{18}$ including RCC. ${ }^{19}$ The association of anemia with death from RCC has also been extensively evaluated and accepted by the UICC/AJCC as a valid indicator of prognosis, particularly in the setting of metastatic disease. ${ }^{5}$ However, when patients with all stages of RCC have been considered as a single group, the importance of anemia as a prognostic indicator has been less than clear. $8,20,21$ Our present study, in which preoperative hemoglobin values were available in 1695 of 1707 (99.3\%) consecutive RCC patients, is a significantly larger cohort with a substantially higher proportion of patients who may be evaluated than previously reported studies, and thus minimizes selection bias and adds power to an analysis of preoperative hemoglobin values as a potential prognostic indicator for patients with RCC. Moreover, most patients in our present study exhibited organ-confined disease (pT2 or less RCC; Table 1). In this context, we demonstrate that preoperative anemia serves as an independent prognostic indicator that heralds an increased risk of cancer-specific death for patients with clinically confined clear cell RCC. 
Elevations in ESR are typically associated with inflammatory processes, such as infection, autoimmunity or malignancy, through mechanisms that are poorly understood, but which may involve inflammatory mediators such as fibrinogen or interleukin- $6 .{ }^{22} \mathrm{RCC}$ is often associated with elevations in ESR, even in the setting of organ-confined diseases, perhaps consequent to interactions between the tumor and host immunity. In addition, most previous studies examining the relationship of ESR with death from RCC have demonstrated a strong association with survival, including a recent study reported by our institution. ${ }^{22}$ The incidence of elevated ESR in patients with RCC has been reported to range from $23 \%$ to $50 \%{ }^{22}$ Using our institution's gender-specific criteria, ESR values were elevated in 297 of 677 (44\%) of patients with available data. As in the case of hypercalcemia and anemia, we report that preoperative elevations in ESR also independently predict an increased risk of cancer-specific death for patients with clinically confined clear cell RCC.

Elevation of serum ALP in the presence of nonmetastatic RCC has previously been reported by Stauffer as one of several biochemical abnormalities that manifest in hepatic dysfunction. 23 The pathophysiology of hepatic dysfunction induced by localized RCC remains poorly understood. ${ }^{16}$ Isolated elevation of ALP in association with RCC may result from ectopic production of ALP by tumor cells. ${ }^{24}$ ALP has been reported to be univariately associated with cancer-specific survival among patients with RCC in recent studies ${ }^{5,8}$ In our series, however, ALP was not associated with death from RCC either univariately or after multivariate adjustment. Coupled with recent reports demonstrating a lack of association on multivariate analysis between elevated ALP and death from RCC, ${ }^{5,8}$ our results suggest that serum ALP is not a significant prognostic factor for patients with localized RCC. Nevertheless, it remains our practice to obtain serum ALP measurements to facilitate the assessment of patients with newly diagnosed RCC to determine whether bone scintigraphy is indicated to rule out the presence of concomitant metastatic disease.

Limitations of our present study include its retrospective nature and difficulties associated with comparing our data against previous studies that have employed different definitions of abnormal pre-perative blood values. In addition, serum calcium and ESR values were absent for $317(18.6 \%)$ and $1030(60.3 \%)$ of the 1707 patients in our study, respectively. Yet, these proportions are comparable to what has been reported in prior studies. ${ }^{8,22}$ In fact, a significantly higher proportion of patients in our study were available for analysis of preoperative anemia as a prognostic indicator in clinically localized RCC. ${ }^{8}$ Given that we restricted our analyses to patients with clinically confined, clear cell RCC, we believe that we minimized the potential for confounding bias that might emanate from combining localized and advanced RCC patients into one group or combining all RCC histologic subtypes into one pathologic entity.

Specifically, bias from adjuvant therapy and treatment with palliative intent was minimized by this approach. Yet, even after limiting our analysis to patients with clinically localized clear cell RCC, our study is still significantly larger than prior studies reported, $5,8,17,20,24$ thereby permitting multivariate adjustments for primary tumor classification, tumor size, nuclear grade, coagulative tumor necrosis, and ECOG performance status.

\section{Conclusions}

Preoperative hypercalcemia, anemia, and elevated ESR portend an increased risk for cancerspecific death in patients with clinically confined clear cell RCC. These associations remain statistically significant after multivariate adjustment for commonly employed prognostic features including primary tumor classification, tumor size, nuclear grade, coagulative tumor necrosis, and ECOG performance status. Inclusion of these variables in future models may improve prognostic accuracy. We believe these factors should be routinely assessed and included in prospective studies of outcome in RCC patients. 


\section{References}

1. Jemal A, Murray T, Ward E, et al. Cancer statistics, 2005. CA Cancer J Clin 2005;55:10-30. [PubMed: 15661684]

2. Leibovich BC, Blute ML, Cheville JC, et al. Prediction of progression after radical nephrectomy for patients with clear cell renal cell carcinoma: a stratification tool for prospective clinical trials. Cancer 2003;97:1663-1671. [PubMed: 12655523]

3. Srigley JR, Hutter RV, Gelb AB, et al. Current prognostic factors: renal cell carcinoma: Workgroup No. 4. Union Internationale Contre le Cancer (UICC) and the American Joint Committee on Cancer (AJCC). Cancer 1997;80:994-6. [PubMed: 9307206]

4. Gelb AB. Renal cell carcinoma: current prognostic factors. Union Internationale Contre le Cancer (UICC) and the American Joint Committee on Cancer (AJCC). Cancer 1997;80:981-986. [PubMed: 9307202]

5. Motzer RJ, Mazumdar M, Bacik J, et al. Survival and prognostic stratification of 670 patients with advanced renal cell carcinoma. J Clin Oncol 1999;17:2530-2540. [PubMed: 10561319]

6. Zisman A, Pantuck AJ, Dorey F, et al. Improved prognostication of renal cell carcinoma using an integrated staging system [see comment]. J Clin Oncol 2001;19:1649-1657. [PubMed: 11250993]

7. Frank I, Blute ML, Cheville JC, et al. An outcome prediction model for patients with clear cell renal cell carcinoma treated with radical nephrectomy based on tumor stage, size, grade and necrosis: the SSIGN score. J Urol 2002;168:2395-2400. [PubMed: 12441925]

8. Kim HL, Belldegrun AS, Freitas DG, et al. Paraneoplastic signs and symptoms of renal cell carcinoma: implications for prognosis. J Urol 2003;170:1742-1746. [PubMed: 14532767]

9. Cheville JC, Lohse CM, Zincke H, et al. Comparisons of outcome and prognostic features among histologic subtypes of renal cell carcinoma. Am J Surg Pathol 2003;27:612-624. [PubMed: 12717246]

10. Furge KA, Lucas KA, Takahashi M, et al. Robust classification of renal cell carcinoma based on gene expression data and predicted cytogenetic profiles. Cancer Res 2004;64:4117-4121. [PubMed: 15205321]

11. Junker K, Weirich G, Amin MB, et al. Genetic subtyping of renal cell carcinoma by comparative genomic hybridization. Recent Res Cancer Res 2003;162:169-175.

12. Sengupta S, Lohse CM, Leibovich BC, et al. Histologic coagulative tumor necrosis as a prognostic indicator of renal cell carcinoma aggressiveness. Cancer 2005;104:511-520. [PubMed: 15973740]

13. Storkel S, Eble JN, Adlakha K, et al. Classification of renal cell carcinoma: Workgroup No. 1. Union Internationale Contre le Cancer (UICC) and the American Joint Committee on Cancer (AJCC). Cancer 1997;80:987-989. [PubMed: 9307203]

14. Kovacs G, Akhtar M, Beckwith BJ, et al. The Heidelberg classification of renal cell tumours. J Pathol 1997;183:131-133. [PubMed: 9390023]

15. Frank I, Blute ML, Cheville JC, et al. A multifactorial postoperative surveillance model for patients with surgically treated clear cell renal cell carcinoma [see comment]. J Urol 2003;170:2225-2232. [PubMed: 14634384]

16. Gold PJ, Fefer A, Thompson JA. Paraneoplastic manifestations of renal cell carcinoma. Semin Urol Oncol 1996;14:216-222. [PubMed: 8946620]

17. Fahn HJ, Lee YH, Chen MT, et al. The incidence and prognostic significance of humoral hypercalcemia in renal cell carcinoma. J Urol 1991;145:248-2450. [PubMed: 1988711]

18. Spivak JL. The anaemia of cancer: death by a thousand cuts. Nat Rev Cancer 2005;5:543-555. [PubMed: 15965494]

19. Pantuck AJ, Zeng G, Belldegrun AS, et al. Pathobiology, prognosis, and targeted therapy for renal cell carcinoma: exploiting the hypoxia-induced pathway. Clin Cancer Res 2003;9:4641-4652. [PubMed: 14581333]

20. Nacey JN, Delahunt B. Renal cell carcinoma: I. Clinical indicators of prognosis. N Z Med J 1986;99:531-533. [PubMed: 3461393]

21. Sene AP, Hunt L, McMahon RF, et al. Renal carcinoma in patients undergoing nephrectomy: analysis of survival and prognostic factors. Br J Urol 1992;70:125-134. [PubMed: 1393433] 
22. Sengupta S, Lohse CM, Cheville JC, et al. The preoperative erythrocyte sedimentation rate is an independent prognostic factor in renal cell carcinoma. Cancer 2006;106:304-312. [PubMed: 16353202]

23. Stauffer MH. Nephrogenic hepatosplenomegaly. Gastroenterology 1961;40:694-695.

24. Chuang YC, Lin AT, Chen KK, et al. Paraneoplastic elevation of serum alkaline phosphatase in renal cell carcinoma: incidence and implication on prognosis. J Urol 1997;158:1684-1687. [PubMed: 9334578] 
Table 1

Summary of clinical and pathologic features for 1707 patients with clinically confined clear cell renal cell carcinoma

\begin{tabular}{lc}
\hline Feature & $\mathbf{N}(\mathbf{\%})$ \\
\hline ECOG performance status $(\mathrm{N}=1122)$ & $1019(90.8)$ \\
0 & $103(9.2)$ \\
$\geq 1$ & \\
Primary tumor size & $534(31.3)$ \\
$<5 \mathrm{~cm}$ & $1173(68.7)$ \\
$\geq 5 \mathrm{~cm}$ & \\
2002 primary tumor classification & $397(23.3)$ \\
pT1a & $464(27.2)$ \\
pT1b & $364(21.3)$ \\
pT2 & $137(8.0)$ \\
pT3a & $323(18.9)$ \\
pT3b & $13(0.8)$ \\
pT3c & $9(0.5)$ \\
pT4 & $188(11.0)$ \\
Nuclear grade & $812(47.6)$ \\
1 & $616(36.1)$ \\
2 & $91(5.3)$ \\
4 & $1289(75.5)$ \\
Coagulative tumor necrosis & $418(24.5)$ \\
No & \\
Yes & \\
\hline
\end{tabular}




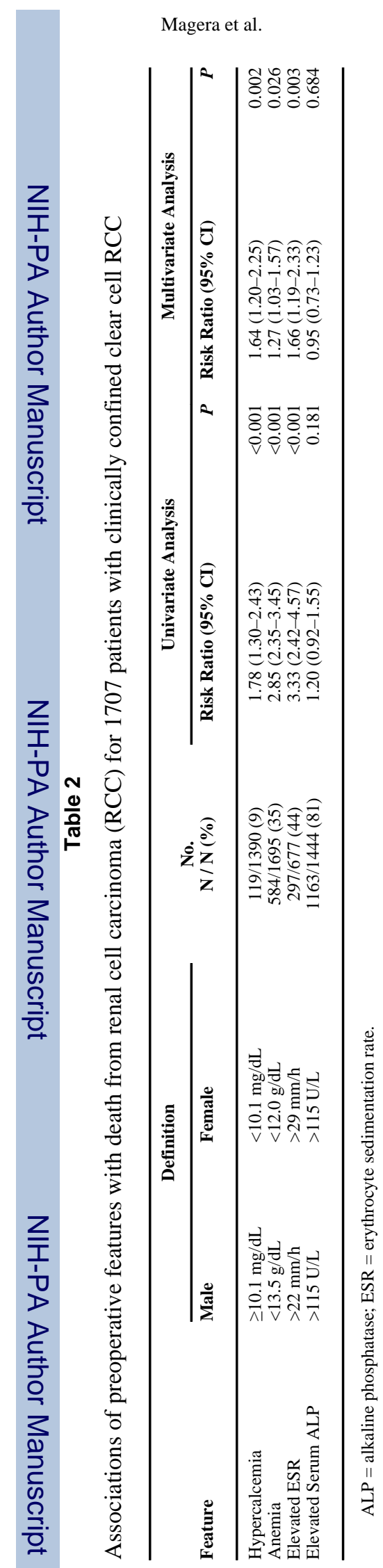

Urology. Author manuscript; available in PMC 2009 December 7. 